\title{
Study on storage stability of grapes juice preserved with sodium benzoate, potassium sorbate and potassium metabisulphite
}

Sumayya Rani ${ }^{*}$, Jamal Abdul Nasir ${ }^{2}$, Muhammad Ayub ${ }^{2}$, Uzma Shahni ${ }^{3}$ and Alam Zeb ${ }^{2}$

1. Department of Agriculture (Food Science \& Technology), The University of Swabi, Anbar Swabi-Pakistan

2. Department of Food Science \& Technology, The University of Agriculture Peshawar-Pakistan

3. Department of Food\& Nutrition, Abdul WaliKhan University Mardan-Pakistan

*Corresponding author's email: Sumayya@uoswabi.edu.pk

Citation

Sumayya Rani, Jamal Abdul Nasir, Muhammad Ayub, Uzma Shahni and Alam Zeb. Study on storage stability of grape juice preserved with sodium benzoate, potassium sorbate and potassium metabisulphite. Pure and Applied Biology. Vol. 7, Issue 1, pp103-111. http://dx.doi.org/10.19045/bspab.2018.70013

\begin{tabular}{llll}
\hline \hline Received: 29/09/2017 & Revised: 27/12/2017 & Accepted: 31/12/2017 & Online First: 10/01/2018 \\
\hline \hline
\end{tabular}

\section{Abstract}

This study was carried out to find the effect of potassium metabisulphite, potassium sorbate and sodium benzoate on the preservation and overall quality of grapes juice. Total seven samples were made with this juice which were named as $\mathbf{J}_{0}, \mathbf{J}_{1}, \mathbf{J}_{2}, \mathbf{J}_{3}, \mathbf{J}_{4}, \mathbf{J}_{5}, \mathbf{J}_{6}$. They were kept in plastic bottles and stored at room temperature. All samples were analyzed at fifteen days interval physiochemically ( $\mathrm{pH}$, total soluble solids, $\%$ acidity, ascorbic acid, reducing and non-reducing sugar) and organoleptically (color, flavor, taste and overall acceptability) for a total period of 90 days. Results obtained from statistical analysis $(\alpha<0.05)$ showed that storage interval and preservation had significant effect on physiochemical and organoleptic characteristics. Total soluble solids increased maximum in $\mathrm{J}_{0}(7.46 \%)$ and minimum in $\mathrm{J}_{4}(2.79 \%)$. Ascorbic acid reduced maximum in $\mathrm{J}_{0}(70.40 \%)$ and minimum in $\mathbf{J}_{2}(29.14 \%)$. $\mathrm{pH}$ reduced maximum in $\mathbf{J}_{0}(17.23 \%)$ and minimum in $\mathrm{J}_{4}(4.06 \%)$. Acidity increased maximum in $\mathrm{J}_{0}(41.00 \%)$ and minimum in $\mathrm{J}_{4}(11.18 \%)$. Reducing sugars increased maximum in $\mathrm{J}_{0}(69.51 \%)$ and minimum in $\mathrm{J}_{4}(8.81 \%)$. Non-reducing sugars decreased maximum in $\mathrm{J}_{4}(83.33 \%)$ and minimum in $\mathrm{J}_{2}(36.22 \%)$. Color score decreased maximum for $\mathrm{J}_{1}(53.30 \%)$ and minimum for $\mathrm{J}_{4}(8.00 \%)$. Flavor score decreased maximum for $\mathrm{J}_{2}(50 \%)$ and minimum for $\mathrm{J}_{6}(12.43 \%)$. Taste score decreased maximum for $\mathrm{J}_{2}(62.66 \%)$ and minimum for $\mathrm{J}_{4}(8.00 \%)$. Overall acceptability score decreased maximum for $\mathrm{J}_{2}(54.35 \%)$ and minimum for $\mathrm{J}_{4}(10.26 \%)$. $\mathrm{J}_{4}$ [(grapesjuice + potassium metabisulphite $(0.05 \%)$ + potassium sorbate $(0.05 \%)$ ] remained very acceptable during storage while $\mathrm{J}_{0}$ [grapes juice + no preservative] was found very bad. Future study is recommonded on microflora assesment, micronutrients assesment, flavonoids and polyphenols extraction which can be added to other food products to prevent cancer in population.

Keywords: Acidity; Ascorbic acid; Organoleptics; $\mathrm{pH}$; Sodium benzoate; Total soluble solids

\section{Introduction}

Grapes (Vitis vinifera) are belonging to group of berries because of having tiny size and soft structure. They are members of kingdom plantae and genera vitis.s Grapes were actually originated in Turkey. History also have evidences of their presenc in Egypt, Greeks, Europe, North Africa and North 
America [1]. Throughout the world grapes are grown in different countries. According to FAO reporting its world wise yield from $75,866 \mathrm{~km}^{2}$ was $71 \%$ and in the dried farm consumption was recorded $2 \%$ of total produced [2].Grapes juice is ever liking product which is directly use for drinking and sometimes it is converted into other products. It is also fermented to produce wines and vinegar. It consists of $89 \%$ water having large concentration of glucose and fructose. The acidity content $f$ grapes juice is high because of the presence of tartaric acid, maleic acid and ascorbic acid. They also give a wide range of antioxidants such as flavonoids, anthocyanins, geranial, linalool, nerols and tennins. These antioxidants are responsible for preventing different types of cancers in the body [3]. Food graded additives such as benzoate, sorbate etc are added to juices in order to increase their shelf life [4]. In juices benzoate is used up to $0.1 \%$ for preservation which is recommended amount in juices but some countries have permission for up to $0.15 \%$ to $0.25 \%$. Sorbic acid such as sorbates are widely used as preservative in juices which act as antimicrobial agent to control microorganisms in food and food products [5]. It is effective if it is used in the range of 0.03-0.05\%. Sorbates are used in the products like jam, jellies while benzoic acid is used in the fruits products such as juices, squashes as they already have acidic $\mathrm{pH}$. It is permitted in the range of $0.01-0.02 \%$ which is effective range in these products [6].Grapes are very important berry fruit but are highly perishable and have very short shelf life [7]. During peak season they are produced in abundance but due to improper storage and handling facilities their losses occur. They are processed through different technical application to different products such as juice, squashes, syrup and jam to make their availability possible at any season of the year. Beverages are liked by consumers in the hot weather. Usually citrus fruits are used for extracting juices but with the emerging technologies new fruits are also utilized to produce consumer oriented products in every season. To give satisfaction to the taste buds of diabetic patients, different juices are prepared with non-nutritive sweetners [8]. Keeping in mind the rising demands of the product, nutritional significance and recommendations of health professionals, this study was designed. This study aimed to evaluate the effect of sodium benzoate, potassium sorbate, potassium metabisulphite and storage duration on keeping quality of grapes juice.

\section{Materials and methods}

Good quality grapes were purchased from local market of Peshawar and were brought to food processing and analytical Laboratory of The University of Agriculture, Peshawar. After washing cleaning and stemming juice was extracted with the help of electric juicer and was filtered with muslin cloth. Total seven samples were made with concentrations of sodium benzoate, potassium sorbate and potassium metabisulphite, as shown in (Table 1).

Table 1. Sample plan

\begin{tabular}{|c|c|}
\hline $\mathrm{J}_{0}$ & Grapes juice + no preservative \\
\hline $\mathrm{J}_{1}$ & $\begin{array}{l}\text { Grapes juice }+ \text { Potassium } \\
\text { metabisulphite }(0.1 \%)\end{array}$ \\
\hline $\mathbf{J}_{2}$ & $\begin{array}{l}\text { Grapes juice + Sodium benzoate } \\
(0.1 \%)\end{array}$ \\
\hline $\mathbf{J}_{3}$ & $\begin{array}{l}\text { Grapes juice + potassium sorbate } \\
(0.1 \%\end{array}$ \\
\hline $\mathrm{J}_{4}$ & $\begin{array}{l}\text { Grapes juice }+ \text { Potassium } \\
\text { metabisulphite }(0.05 \%)+ \\
\text { Potassium sorbate }(0.05 \%)\end{array}$ \\
\hline $\mathrm{J}_{5}$ & $\begin{array}{l}\text { Grapes juice }+ \text { Potassium } \\
\text { metabisulphitem }(0.05 \%)+ \\
\text { Sodium benzoate }(0.05 \%)\end{array}$ \\
\hline $\mathrm{J}_{6}$ & $\begin{array}{l}\text { Grapes juice }+ \text { potassium sorbate } \\
(0.05)+\text { Sodium benzoate }(0.05)\end{array}$ \\
\hline
\end{tabular}

All samples were stored in plastic bottles at room temperature. They were analysed physiochemically $(\mathrm{pH}$, total soluble solids, 
acidity, ascorbic acid, reducing sugars and non-reducing sugar) and organoleptically (color, flavor, taste and overall acceptability) for total period of 90 days at 15 days interval. Physiochemical Analysis

All the samples were analyzed physiochemically for $\mathrm{pH}$, total soluble solids, $\%$ acidity Ascorbic acid, reducing sugars and non-reducing sugars according to the standard methods of [9].

\section{Organoleptic evaluation}

All samples were presented to a panel of ten trained judges and were analyzed for color, flavor, taste and overall acceptability using 9 points hedonic scale as was described by [10].

\section{Statistical Analysis}

Data was statistically analyzed by CRD 2 factorial design as recommended by [11] and means were separated by applying least significant difference (LSD) test at $\alpha(<0.05)$ probability level as defined by [12].

\section{Results and discussion}

Ascorbic acid (mg/ 100g) of grapes juice samples

There was a gradual decrease in ascorbic acid content of samples during storage. The average ascorbic acid value was decreased from 25.8 to 12.5 at $\alpha$ value less than 0.05 . Maximum average value was observed in $\mathrm{J}_{5}$ (22.9) and minimum in $J_{2}$ (16.4). Highest percent decrease in ascorbic acid content was observed in $\mathrm{J}_{0}(70.40 \%)$ and lowest in $\mathrm{J}_{2}$ $(39.27 \%)$ as shown in (Table 2). Decrease in ascorbic acid content was also reported by [13] in his work on apple juice samples, by [14] in grape juice samples, by [15] in apple pulp samples, by [16] in apple juice samples, by [17] in kinnow juice samples, and by [18] in mango leather samples.

Table 2. Effect on ascorbic acid content of grapes juice samples

\begin{tabular}{|c|c|c|c|c|c|c|c|c|c|}
\hline & \multicolumn{7}{|c|}{ Storage intervals (Days) } & \multirow{2}{*}{ \% Decrease } & Average \\
\cline { 2 - 10 } Treatments & Fresh & 15 & 30 & 45 & 60 & 75 & 90 & 70.4 & $16.4 \mathrm{f}$ \\
\hline $\mathrm{J}_{0}$ & 25.2 & 22.4 & 19.0 & 16.0 & 13.0 & 10.0 & 7.3 & 7.15 & $17.7 \mathrm{~d}$ \\
\hline $\mathrm{J}_{1}$ & 25.1 & 23.8 & 20.1 & 18.3 & 14.9 & 12.2 & 9.5 & 62.15 & $17.5 \mathrm{e}$ \\
\hline $\mathrm{J}_{2}$ & 24.7 & 22.6 & 20.5 & 17.4 & 15.2 & 12.0 & 10.1 & 59.10 & $21.8 \mathrm{~b}$ \\
\hline $\mathrm{J}_{3}$ & 27.2 & 25.5 & 24.2 & 22.0 & 20.8 & 18.6 & 14.4 & 47.05 & $20.0 \mathrm{a}$ \\
\hline $\mathrm{J}_{4}$ & 24.7 & 22.5 & 21.5 & 20.3 & 19.0 & 17.2 & 15.0 & 39.27 & 20.0 \\
\hline $\mathrm{J}_{5}$ & 27.1 & 25.7 & 24.5 & 22.3 & 20.8 & 17.2 & 16.0 & 40.95 & $22.9 \mathrm{c}$ \\
\hline $\mathrm{J}_{6}$ & 27.0 & 25.7 & 22.5 & 22.5 & 21.2 & 18.5 & 15.7 & 41.85 & $21.8 \mathrm{c}$ \\
\hline Average & $25.8 \mathrm{~d}$ & $24.0 \mathrm{~d}$ & $25.3 \mathrm{c}$ & $19.9 \mathrm{~b}$ & $17.97 \mathrm{~b}$ & $15.1 \mathrm{a}$ & $12.5 \mathrm{a}$ & & \\
\hline
\end{tabular}

\section{pH of grapes juice samples}

There was a gradual decrease in $\mathrm{pH}$ content of samples. The average $\mathrm{pH}$ value was decreased from 4.10 to 3.71 at $\alpha$ value less than 0.05. Maximum average value was observed in $\mathrm{J}_{4}$ (4.24) and minimum in $\mathrm{J}_{0}$ (3.76). Highest $\%$ decrease in $\mathrm{pH}$ content was observed in $\mathrm{J}_{0}(17.23 \%)$ and minimum in $\mathrm{J}_{4}$ $(4.06 \%)$ as shown in (Table 3). Decrease in $\mathrm{pH}$ during storage was also reported by [19] in guava pulp samples, by [20] in strawberry juice samples, by [21] in pineapple leather samples and by [22] in cashew apple juice samples.

\section{Total soluble solids ( ${ }^{\circ}$ Brix) of grapes juice} samples

There was a gradual increase in total soluble solids content of samples. The average value was increased from 18.42 to 19.31 at $\alpha$ value less than 0.05. Maximum average value was observed in $\mathrm{J}_{4}(21.84)$ and minimum in $\mathrm{J}_{0}$ (15.04). Highest \% increase in total soluble solids content was observed in $\mathrm{J}_{0}(7.46 \%)$ and minimum in $\mathrm{J}_{4}(2.79 \%)$ as shown in (Table 4). These results are in agreements with the 
findings of [23] in mango pulp samples. The same slow increase in total soluble solids content was also shown by [24] in their

\begin{tabular}{|c|c|c|c|c|c|c|c|c|c|}
\hline \multirow[b]{2}{*}{ Treatments } & \multicolumn{7}{|c|}{ Storage intervals (Days) } & \multirow[b]{2}{*}{ \% Decrease } & \multirow[b]{2}{*}{ Average } \\
\hline & Fresh & 15 & 30 & 45 & 60 & 75 & 90 & & \\
\hline $\mathrm{J}_{0}$ & 4.12 & 4.12 & 4.07 & 3.62 & 3.52 & 3.49 & 3.41 & 17.23 & $3.76 \mathrm{e}$ \\
\hline $\mathrm{J}_{1}$ & 4.08 & 4.09 & 3.84 & 3.95 & 3.83 & 3.81 & 3.75 & 8.08 & $3.90 \mathrm{~cd}$ \\
\hline $\mathrm{J}_{2}$ & 4.07 & 4.12 & 4.05 & 3.95 & 3.89 & 3.82 & 3.79 & 6.87 & $3.95 b c$ \\
\hline $\mathrm{J}_{3}$ & 4.11 & 4.14 & 4.11 & 4.03 & 3.97 & 3.91 & 3.82 & 7.05 & $4.01 \mathrm{~b}$ \\
\hline $\mathrm{J}_{4}$ & 4.18 & 4.45 & 4.37 & 4.28 & 4.21 & 4.19 & 4.01 & 4.06 & $4.24 \mathrm{a}$ \\
\hline $\mathrm{J}_{5}$ & 4.04 & 4.06 & 4.00 & 3.90 & 3.70 & 3.69 & 3.63 & 10.14 & $3.86 \mathrm{cde}$ \\
\hline $\mathrm{J}_{6}$ & 4.12 & 4.00 & 3.95 & 3.76 & 3.71 & 3.65 & 3.59 & 12.86 & $3.82 \mathrm{de}$ \\
\hline Average & $4.10 \mathrm{a}$ & $4.14 \mathrm{a}$ & $4.05 \mathrm{a}$ & $3.92 b$ & $3.83 b c$ & $3.79 \mathrm{~cd}$ & $3.71 \mathrm{~d}$ & 9.51 & \\
\hline
\end{tabular}

Table 4. Effect on total soluble solids of grapes juice samples

\begin{tabular}{|c|c|c|c|c|c|c|c|c|c|}
\hline \multirow{2}{*}{ Treatments } & \multicolumn{7}{|c|}{ Storage intervals (Days) } & \multirow{2}{*}{$\begin{array}{c}\% \\
\text { Increase }\end{array}$} & \multirow{2}{*}{ Average } \\
\hline & Fresh & 15 & 30 & 45 & 60 & 75 & 90 & & \\
\hline $\mathrm{J}_{0}$ & 14.5 & 14.5 & 15.0 & 15.1 & 15.2 & 15.5 & 15. & 7.46 & $15.07 \mathrm{f}$ \\
\hline $\mathrm{J}_{1}$ & 19.0 & 19.0 & 19.5 & 19.6 & 19.8 & 19.8 & 19.9 & 4.13 & $19.51 \mathrm{~d}$ \\
\hline $\mathrm{J}_{2}$ & 15.5 & 15.5 & 15.6 & 15.8 & 16.0 & 16.2 & 16.2 & 4.51 & $15.82 \mathrm{e}$ \\
\hline $\mathrm{J}_{3}$ & 19.5 & 19.6 & 20.0 & 20.2 & 20.2 & 20.5 & 20.7 & 6.15 & $20.1 \mathrm{~b}$ \\
\hline $\mathrm{J}_{4}$ & 21.5 & 21.7 & 21.7 & 21.9 & 21.9 & 22.1 & 22.1 & 2.79 & $21.84 \mathrm{a}$ \\
\hline $\mathrm{J}_{5}$ & 19.5 & 19.7 & 20.0 & 20.0 & 20.0 & 20.1 & 20.1 & 3.07 & $19.91 \mathrm{c}$ \\
\hline $\mathrm{J}_{6}$ & 19.5 & 19.5 & 19.5 & 19.7 & 20.0 & 20.2 & 20.5 & 5.12 & $19.84 \mathrm{c}$ \\
\hline Average & $18.42 \mathrm{~d}$ & $18.5 \mathrm{~d}$ & $18.75 \mathrm{c}$ & $18.9 \mathrm{~b}$ & $19.01 \mathrm{~b}$ & $19.2 \mathrm{a}$ & $19.31 \mathrm{a}$ & & \\
\hline
\end{tabular}

Titratable acidity (\%) of grapes juice samples

There was a gradual increase in acidity content of samples. The average value was increased from 1.09 to 1.32 at $\alpha$ value less than 0.05 . Maximum average value was observed in $\mathrm{J}_{5}(1.69)$ and minimum in $\mathrm{J}_{2}(0.92)$. Highest spinach orange carrot juice samples and by [25] in lime juice samples.

Table 5. Effect on titratable acidity (\%) of grapes juice samples

\begin{tabular}{|c|c|c|r|r|r|r|r|c|c|}
\hline \multirow{2}{*}{ Treatments } & \multicolumn{7}{|c|}{ Storage intervals (Days) } & \%Increa & \multirow{2}{*}{ Average } \\
\cline { 2 - 10 } & Fresh & 15 & 30 & 45 & 60 & 75 & 90 & & \\
\hline $\mathrm{J}_{0}$ & 0.90 & 1.0 & 1.71 & 1.97 & 1.24 & 1.31 & 1.41 & 41.00 & $0.98 \mathrm{e}$ \\
\hline $\mathrm{J}_{1}$ & 1.00 & 1.02 & 1.06 & 1.09 & 1.09 & 1.11 & 1.13 & 13.00 & $1.07 \mathrm{~d}$ \\
\hline $\mathrm{J}_{2}$ & 0.84 & 0.88 & 0.90 & 0.91 & 0.95 & 0.98 & 1.01 & 20.23 & $0.92 \mathrm{f}$ \\
\hline $\mathrm{J}_{3}$ & 1.00 & 1.05 & 1.10 & 1.13 & 1.20 & 1.23 & 1.23 & 23.00 & $1.13 \mathrm{c}$ \\
\hline $\mathrm{J}_{4}$ & 1.43 & 1.43 & 1.51 & 1.55 & 1.57 & 1.57 & 1.59 & 11.18 & $1.52 \mathrm{~b}$ \\
\hline $\mathrm{J}_{5}$ & 1.49 & 1.60 & 1.65 & 1.70 & 1.78 & 1.82 & 1.88 & 26.17 & $1.69 \mathrm{a}$ \\
\hline $\mathrm{J}_{6}$ & 1.03 & 1.07 & 1.11 & 1.17 & 1.21 & 1.23 & 1.27 & 23.30 & $1.15 \mathrm{c}$ \\
\hline Average & $1.09 \mathrm{e}$ & $1.12 \mathrm{e}$ & $1.71 \mathrm{a}$ & $1.21 \mathrm{~d}$ & $1.25 \mathrm{c}$ & $1.29 \mathrm{~b}$ & $1.32 \mathrm{~b}$ & & \\
\hline
\end{tabular}


Reducing sugar of grapes juice samples. There was a gradual increase in reducing sugars content of samples. The average value was increased from 11.0 to 13.35 at $\alpha$ value less than 0.05. Maximum average value was observed in $\mathbf{J}_{1}$ (13.55) and minimum in $\mathbf{J}_{2}$ (9.90). Highest \% increase in reducing sugars content was observed in $\mathrm{J}_{0}(69.51 \%)$ and minimum in $\mathrm{J}_{4}(8.81 \%)$ as shown in (Table 6). The same increase in reducing sugars content was reported by [28] in strawberry juice samples and by [29] on dehydrated mango slices

Table 6. Effect on reducing sugar of grapes juice samples

\begin{tabular}{|c|c|c|c|c|c|c|c|c|c|}
\hline & \multicolumn{7}{|c|}{ Storage intervals (Days) } & \multirow{2}{*}{ \% Increase } & Average \\
\cline { 2 - 10 } Treatments & Fresh & 15 & 30 & 45 & 60 & 75 & 90 & $13.70 \mathrm{c}$ \\
\hline $\mathrm{J}_{0}$ & 8.20 & 8.45 & 12.20 & 12.50 & 12.95 & 13.50 & 13.90 & 69.51 & $13.55 \mathrm{a}$ \\
\hline $\mathrm{J}_{1}$ & 11.90 & 12.20 & 12.92 & 13.21 & 14.22 & 14.90 & 15.50 & 30.25 & 13.920 \\
\hline $\mathrm{J}_{2}$ & 9.00 & 9.21 & 9.42 & 9.80 & 10.20 & 10.50 & 11.00 & 22.22 & $9.90 \mathrm{~d}$ \\
\hline $\mathrm{J}_{3}$ & 11.11 & 11.70 & 12.00 & 12.52 & 12.70 & 12.95 & 13.50 & 21.57 & $12.35 \mathrm{bc}$ \\
\hline $\mathrm{J}_{4}$ & 12.82 & 12.92 & 13.20 & 13.50 & 13.79 & 13.80 & 13.95 & 8.81 & $13.40 \mathrm{a}$ \\
\hline $\mathrm{J}_{5}$ & 12.20 & 12.42 & 12.62 & 12.80 & 12.95 & 13.20 & 13.40 & 9.83 & $12.80 \mathrm{ab}$ \\
\hline $\mathrm{J}_{6}$ & 11.11 & 11.42 & 11.62 & 11.79 & 11.86 & 11.95 & 12.20 & 9.81 & $11.70 \mathrm{c}$ \\
\hline Average & $11.00 \mathrm{e}$ & $11.18 \mathrm{de}$ & $11.90 \mathrm{~cd}$ & $12.30 \mathrm{bc}$ & $12.66 \mathrm{abc}$ & $12.90 \mathrm{ab}$ & $13.35 \mathrm{a}$ & & \\
\hline
\end{tabular}

\section{Non-reducing sugar}

There was a gradual decrease in nonreducing sugars content of samples. The average value was decreased from 2.39 to 1.27 at $\alpha$ value less than 0.05. Maximum average value was observed in $J_{2}$ (2.96) and minimum in $\mathrm{J}_{0}(0.55)$. Highest $\%$ decrease in non-reducing sugars content was observed in $\mathrm{J}_{4}(83.33 \%)$ and minimum in $\mathrm{J}_{2}(36.22 \%)$ as shown in (Table 7). The same decreasing trend in non-reducing sugars content was also shown by [30] in melon cube samples treated with non-caloric sweetners and by [31] in kinnow juice samples.

Table 7. Effect on non-reducing sugars of grapes juice samples

\begin{tabular}{|c|c|c|c|c|c|c|c|c|c|}
\hline \multirow{2}{*}{ Treatments } & \multicolumn{7}{|c|}{ Storage intervals (Days) } & \multirow{2}{*}{ Average } & \multirow{2}{*}{ \% Decrease } \\
\cline { 2 - 9 } & Fresh & 15 & 30 & 45 & 60 & 75 & 90 & & \\
\hline $\mathrm{J}_{0}$ & 1.03 & 0.93 & 0.50 & 0.30 & 0.12 & 0.40 & 0.55 & $0.547 \mathrm{e}$ & 46.60 \\
\hline $\mathrm{J}_{1}$ & 3.18 & 2.60 & 2.58 & 2.31 & 2.24 & 2.20 & 2.00 & $2.44 \mathrm{~b}$ & 37.10 \\
\hline $\mathrm{J}_{2}$ & 3.92 & 3.15 & 3.04 & 3.01 & 2.61 & 2.50 & 2.50 & $2.96 \mathrm{a}$ & 36.22 \\
\hline $\mathrm{J}_{3}$ & 1.60 & 1.48 & 1.38 & 1.30 & 1.10 & 0.80 & 0.80 & $1.20 \mathrm{~d}$ & 50.00 \\
\hline $\mathrm{J}_{4}$ & 1.20 & 1.15 & 1.00 & 0.62 & 0.57 & 0.32 & 0.20 & $0.72 \mathrm{e}$ & 83.33 \\
\hline $\mathrm{J}_{5}$ & 1.90 & 1.80 & 1.50 & 1.40 & 1.30 & 1.20 & 1.00 & $1.44 \mathrm{c}$ & 47.36 \\
\hline $\mathrm{J}_{6}$ & 3.90 & 3.40 & 3.10 & 3.29 & 2.59 & 2.20 & 1.90 & $2.91 \mathrm{a}$ & 51.28 \\
\hline Average & $2.39 \mathrm{a}$ & $2.07 \mathrm{~b}$ & $1.87 \mathrm{bc}$ & $1.77 \mathrm{c}$ & $1.50 \mathrm{~d}$ & $1.37 \mathrm{de}$ & $1.27 \mathrm{e}$ & & \\
\hline
\end{tabular}

\section{Colour score of grape juice samples}

There was a gradual decrease in color score of grape juice samples. The average score was decreased from 7.54 to 5.45 at $\alpha$ value less than 0.05. Maximum average score was achieved by $\mathrm{J}_{6}$ (7.60) and minimum by $\mathrm{J}_{0}$
(5.28). Highest \% decrease in color score was observed in $\mathrm{J}_{1}(53.30 \%)$ and minimum by $\mathrm{J}_{4}$ $(8.00 \%)$ as shown in (Table 8). The same decrease in color score was shown by [32] in post-harvest dried samples and by [33] in lime and orange juice samples. 
Table 8. Effect on color score of grapes juice samples

\begin{tabular}{|c|c|c|c|c|c|c|c|c|c|}
\hline \multirow{2}{*}{ Treatments } & \multicolumn{7}{|c|}{ Storage Intervals (Days) } & \multirow{2}{*}{ Average } & \multirow{2}{*}{$\begin{array}{c}\% \\
\text { Decrease }\end{array}$} \\
\hline & Fresh & 15 & 30 & 45 & 60 & 75 & 90 & & \\
\hline $\mathbf{J}_{0}$ & 7.30 & 6.5 & 5.5 & 5.0 & 4.5 & 4.2 & 4.0 & $5.28 \mathrm{c}$ & 45.20 \\
\hline $\mathrm{J}_{1}$ & 7.5 & 6.8 & 6.0 & 5.5 & 5.0 & 4.6 & 3.5 & $5.55 c$ & 53.30 \\
\hline $\mathrm{J}_{2}$ & 7.0 & 6.5 & 6.2 & 5.5 & 5.0 & 4.2 & 3.4 & $5.40 \mathrm{c}$ & 51.40 \\
\hline $\mathrm{J}_{3}$ & 7.5 & 7.2 & 7.0 & 7.0 & 6.6 & 6.5 & 6.5 & $6.90 \mathrm{~b}$ & 13.33 \\
\hline $\mathrm{J}_{4}$ & 7.5 & 7.2 & 7.10 & 7.10 & 7.00 & 6.90 & 6.90 & 7.10ab & 8.00 \\
\hline $\mathrm{J}_{5}$ & 8.0 & 8.00 & 7.50 & 7.50 & 7.00 & 7.00 & 6.90 & $7.41 \mathrm{ab}$ & 13.75 \\
\hline $\mathrm{J}_{6}$ & 8.0 & 8.00 & 7.90 & 7.50 & 7.40 & 7.40 & 7.00 & $7.60 \mathrm{a}$ & 12.50 \\
\hline Average & $7.54 \mathrm{a}$ & $7.17 \mathrm{ab}$ & $6.74 \mathrm{~b}$ & $5.65 \mathrm{c}$ & $5.35 \mathrm{c}$ & $5.22 \mathrm{c}$ & $5.45 \mathrm{c}$ & & \\
\hline
\end{tabular}

Flavor score of grape juice samples

There was a gradual decrease in flavor score of samples. The average score was decreased from 7.30 to 5.58 at $\alpha$ value less than 0.05 . Maximum average score was achieved by $\mathrm{J}_{6}$ (7.25) and minimum by $\mathrm{T}_{1}$ (5.52). Highest $\%$ decrease in flavor score was observed in $\mathrm{J}_{2}(50$ $\%)$ and minimum in $\mathrm{J}_{6}(12.43 \%)$ as shown in (Table 9). The same decrease in flavor score was observed by [34] in orange juice samples. The work of [35] in different juice samples are also in accordance with our results. The same decrease in flavor score was reported by [36] in mandrine juice samples.

\section{Taste score of grape juice samples}

There was a gradual decrease in taste score of grapes juice samples. The average score was decreased from 7.62 to 5.18 at $\alpha$ value less than 0.05 . Maximum average score was achieved by $\mathrm{J}_{4}(7.11)$ and minimum by $\mathrm{J}_{2}$ (4.78). Highest \% decrease in taste score was observed in $\mathrm{J}_{2}(62.66 \%)$ and minimum in $\mathrm{J}_{4}$ $(8.00 \%)$ as shown in (Table 10). The same declining trend in taste score were shown by [37] in their study on jack fruit leather samples and by [38] in palmyrah fruit pulp samples.

Overall acceptability score of grape juice samples

There was a gradual decrease in overall acceptability score of samples. The average score was decreased from 7.48 to 5.41 at $\alpha$ value less than 0.05 . Maximum average score was achieved by $\mathrm{J}_{6}$ (7.37) and minimum by $\mathrm{J}_{2}$ (5.27). Highest $\%$ decrease in overall acceptability score was observed in $\mathrm{J}_{2}$ $(54.35 \%)$ and minimum in $\mathrm{J}_{4}(10.26 \%)$ as shown in (Table 11). The same declining trend in overall acceptability score were shown by [39] in apricot fruit bar samples, by [40] in orange juice stored in glass bottles and by [41] in guava fruit juice samples.

Table 9.Effect on flavor score of grapes juice samples

\begin{tabular}{|c|c|c|c|c|c|c|c|c|c|}
\hline \multirow{2}{*}{ Treatments } & \multicolumn{7}{|c|}{ Storage Intervals (Days) } & \multirow{2}{*}{ Average } & $\begin{array}{c}\text { \% } \\
\text { Decrease }\end{array}$ \\
\cline { 2 - 9 } & Fresh & 15 & 30 & 45 & 60 & 75 & 90 & & 18.57 \\
\hline $\mathrm{J}_{0}$ & 7.00 & 6.80 & 6.50 & 6.20 & 6.15 & 6.00 & 5.70 & $6.33 \mathrm{c}$ & 44.92 \\
\hline $\mathrm{J}_{1}$ & 6.90 & 6.50 & 6.00 & 5.40 & 5.20 & 4.90 & 3.80 & $5.52 \mathrm{~d}$ & 50.00 \\
\hline $\mathrm{J}_{2}$ & 7.00 & 6.80 & 6.70 & 6.00 & 5.40 & 4.10 & 3.50 & $6.14 \mathrm{c}$ & 15.27 \\
\hline $\mathrm{J}_{3}$ & 7.20 & 7.00 & 6.80 & 6.50 & 6.40 & 6.20 & 6.10 & $6.60 \mathrm{bc}$ & 14.66 \\
\hline $\mathrm{J}_{4}$ & 7.50 & 7.00 & 6.90 & 6.80 & 6.70 & 6.50 & 6.40 & $6.82 \mathrm{ab}$ & 12.98 \\
\hline $\mathrm{J}_{5}$ & 7.70 & 7.20 & 7.00 & 6.90 & 6.90 & 6.80 & 6.70 & $7.02 \mathrm{ab}$ & 12.43 \\
\hline $\mathrm{J}_{6}$ & 7.80 & 7.70 & 7.60 & 7.00 & 6.90 & 6.90 & 6.90 & $7.25 \mathrm{a}$ & \\
\hline Average & $7.30 \mathrm{a}$ & $7.00 \mathrm{ab}$ & $6.78 \mathrm{bc}$ & $6.40 \mathrm{~cd}$ & $6.23 \mathrm{de}$ & $5.91 \mathrm{ef}$ & $5.58 \mathrm{f}$ & & \\
\hline
\end{tabular}


Table 10. Effect on taste score of grapes juice samples

\begin{tabular}{|c|c|c|c|c|c|c|c|c|c|}
\hline \multirow{2}{*}{ Treatments } & \multicolumn{7}{|c|}{ Storage Intervals (Days) } & \multirow{2}{*}{ Average } & \multirow{2}{*}{ \% Decrease } \\
\cline { 2 - 9 } & Fresh & 15 & 30 & 45 & 60 & 75 & 90 & \\
\hline $\mathrm{J}_{0}$ & 7.60 & 6.00 & 6.00 & 5.50 & 4.50 & 3.50 & 3.00 & $5.14 \mathrm{c}$ & 60.00 \\
\hline $\mathrm{J}_{1}$ & 7.60 & 7.00 & 6.50 & 6.00 & 5.80 & 5.40 & 5.00 & $6.18 \mathrm{~b}$ & 34.21 \\
\hline $\mathrm{J}_{2}$ & 7.50 & 6.30 & 5.40 & 4.30 & 4.00 & 3.20 & 2.80 & $4.78 \mathrm{c}$ & 62.66 \\
\hline $\mathrm{J}_{3}$ & 7.60 & 7.00 & 6.50 & 5.80 & 5.50 & 5.40 & 5.00 & $6.11 \mathrm{~b}$ & 34.21 \\
\hline $\mathrm{J}_{4}$ & 7.50 & 7.20 & 7.20 & 7.10 & 7.00 & 6.90 & 6.90 & $7.11 \mathrm{a}$ & 8.00 \\
\hline $\mathrm{J}_{5}$ & 7.80 & 7.50 & 7.00 & 7.00 & 6.80 & 6.70 & 6.60 & $7.05 \mathrm{a}$ & 15.38 \\
\hline $\mathrm{J}_{6}$ & 7.90 & 7.50 & 7.30 & 7.20 & 7.11 & 7.00 & 7.00 & $7.28 \mathrm{a}$ & 11.39 \\
\hline Average & $7.62 \mathrm{a}$ & $6.55 \mathrm{~b}$ & $\begin{array}{c}6.55 \\
\mathrm{~b}\end{array}$ & $\begin{array}{c}6.12 \mathrm{~b} \\
\mathrm{c}\end{array}$ & $\begin{array}{c}5.81 \\
\mathrm{~cd}\end{array}$ & $\begin{array}{c}5.44 \\
\mathrm{~d}\end{array}$ & $\begin{array}{c}5.18 \\
\mathrm{~d}\end{array}$ & & \\
\hline
\end{tabular}

Table 11. Effect on overall acceptability score of grapes juice samples

\begin{tabular}{|l|l|l|l|l|l|l|l|l|l|}
\hline \multirow{2}{*}{ Treatments } & \multicolumn{7}{|c|}{ Storage Intervals (Days) } & \multirow{2}{*}{ Average } & \multirow{2}{*}{ \% Decrease } \\
\cline { 2 - 9 } & Fresh & 15 & 30 & 45 & 60 & 75 & 90 & & \\
\hline $\mathrm{J}_{0}$ & 7.0 & 6.23 & 6.00 & 5.56 & 5.05 & 4.56 & 4.23 & $5.58 \mathrm{c}$ & 41.73 \\
\hline $\mathrm{J}_{1}$ & 7.33 & 6.76 & 6.16 & 5.63 & 5.33 & 4.96 & 4.10 & $5.75 \mathrm{c}$ & 44.06 \\
\hline $\mathrm{J}_{2}$ & 7.16 & 6.53 & 6.10 & 5.26 & 4.80 & 3.83 & 3.23 & $5.27 \mathrm{c}$ & 54.35 \\
\hline $\mathrm{J}_{3}$ & 7.43 & 7.06 & 6.76 & 6.43 & 6.16 & 6.03 & 5.86 & $6.53 \mathrm{~b}$ & 21.13 \\
\hline $\mathrm{J}_{4}$ & 7.50 & 7.13 & 7.06 & 7.00 & 6.90 & 6.76 & 6.73 & $7.01 \mathrm{ab}$ & 10.26 \\
\hline $\mathrm{J}_{5}$ & 7.83 & 7.56 & 7.16 & 7.13 & 6.90 & 6.83 & 6.76 & $7.16 \mathrm{a}$ & 13.66 \\
\hline $\mathrm{J}_{6}$ & 7.90 & 7.73 & 7.60 & 7.23 & 7.13 & 7.10 & 6.96 & $7.37 \mathrm{a}$ & 11.89 \\
\hline Average & $7.48 \mathrm{a}$ & $7.02 \mathrm{ab}$ & $6.69 \mathrm{bc}$ & $6.32 \mathrm{~cd}$ & $6.03 \mathrm{de}$ & $5.72 \mathrm{ef}$ & $5.41 \mathrm{f}$ & & \\
\hline
\end{tabular}

\section{Conclusion and recommendations}

From this study it was concluded that addition of preservatives strongly affect the products shelf life and consumer acceptability. During this study we found $\mathrm{J}_{4}$ [(grapes juice + potassium metabisulphite $(0.05 \%)+$ potassium sorbate $(0.05 \%)$ ] remained very acceptable during storage while $\mathrm{J}_{0}$ [grapes juice + no preservative] was found very bad. Future study is recommended on microflora assesment, micronutrients assesment and flavonoids and polyphenols extraction and can be added to other food products to prevent cancer in population. Further study is recommended on refrigeration temperature and mixing of more than two fruits is also recommended to make the product more nutritious. Nutritional assessment and packaging effect study is also recommended for future work.
Authors' contributions

Conceived and designed the experiments: $M$ Ayub \& JA Nasir, Performed the experiments: S Rani \& JA Nasir, Analyzed the data: U Shahni \& JA Nasir, Contributed materials/ analysis/ tools: A Zeb, U Shahni \& S Rani, Wrote the paper: S Rani \& Shahni.

\section{References}

1. Jaya CW \& Das D (2003). Climatic effects of lopophilic acids and related coumpoundson grapes juice and on its micro nutrients. $J$ Agric Chem 55(4):319-324.

2. Reisch BI, Peterson DV \& Martens MH (2009). Seedless Grapes, in Table Grape Varieties for Cool Climates. Agri Exp Stn 30.

3. Saeed A, Ali J, Hassan S, Abbas S, Javid B, Khan FA \& Siddique M (2014). Quality evaluation of different brands of grape juice available in local market of Peshawar city, 
Khyber Pakhtunkhwa Pakistan. Int J Basic Med Sc \& Pharm 4(1):13-15.

4. Hussain I, Zeb A, ShakirI\& Shah AS (2008). Combined effect of sodium benzoate and potassium sorbate on individual and blended juicesof apricot and apple fruits grown in Azad Jammu and Kashmir. Pak J Nutr 7(1):181-185.

5. Sattar A \& Rehman (1987). Effect of canning and storage on pectinandpectic fractions of kinnow juice. Pak J Sc 4:315323.

6. Combina M, DalceroAM, Varsavsky\&Chulze S (1999).Effects of food preservatives on alternaria alternate growth and tenuazonic acid production. Food additives and contaminants 16(10):433-437.

7. Cerdan TG, Gil MA, Fontanet ARM, Azpilicueta \& Belloso OM (2007). Effects of thermal and non-thermal processing treatments on fatty acids and free amino acids of grape juice. Food Control 18(5):473-479.

8. Lyr MM, Sacks GL \& Zakour IP (2010). Impacts of harvesting and processing conditions on green leaf volatile development and phenolics in Concord grape juice. J Food Sci 75(3):297-304.

9. AOAC (2012).Official Methods of Analysis.The Association of the official Analytical Chemists. Arlingto 17th Ed Chap 17.

10. Larmond E (1977). Laboratory methods for sensory evaluation offood: Research branch Canada dept of Agriculture.

11. Gomez KA\& Gomez AA (1984). Statistical procedures for agricultural research. John Wiley \& Sons.

12. Steel RGD \& Torrie JH (1980). Principles and Procedures of Statistics. Analysis of covariance. Principal and procedure of statistics: A biometrical approach 401437.

13. Mehmood Z, Zeb A, Ayub M, Bibi N, Badshah A \& Ullah I (2008). Effect of pasteurization and chemical preservatives on the quality and shelf stability of apple juice. American J Food Tech 3(2): 147153.

14. Zeb A, Ullah I, Ahmad A, Ali K \&Ayub M (2008). Grape juice preservation with benzoate and sorbate. J Advances in Food Sci 31(1): 17-21.

15. Kinh A, Shearer EH, Dunne CP \& Hoover DG(2001). Preparation and preservation of apple pulp with chemical preservatives and mild heat. J Food Prot 28(6): 111-114.

16. Mehmood Z, Zeb A, Ayub M, Bibi N, Badshah A \& Ullah I (2008). Effect of pasteurization and chemical preservatives on the quality and shelf stability of apple juice. American J Food Tech 3(2): 147153.

17. Sandhu KS \& Singh S (2001). Studies on the factors affecting the physico-chemical and organoleptic properties of kinnow juice. J Food Sci\& Tech 38(3):266-269.

18. Azerdo HMC, Brito ES, Moreira GEG, Farias VL \& Bruno LM (2000). Effect of drying and storage time on the physiochemical prpoerties of mango leather. Int J of Food Sci Tech 41:635-638

19. Imran A, Rafiullah K \& Ayub M (2000). Effect of added sugar at various concentrations on the storage stability of guava pulp. Sarhad J Agric 7(25): 35-39.

20. AyubM, Ullah J, Zeb A \& Muhammad A (2009). Evaluation of strawberry juice preserved with chemical preservatives at refrigeration temperature. I $J \quad N u t$ Metabolism 2(2): 027-032.

21. Phimpherian C, Angchud A, Jangshud K, Therdthai N, Prinyawiwatkul WHK (2011). Physicochemical characteristics and sensory optamization of pineapple leather snack as affected by glucose syrup and pectin concentrations. Int $J$ of Food Sci Tech 46:972-981.

22. Cecilia E \& Maia GA (2002). Storage stability of cashew apple juice preserved by hot fill and aseptic process. Sc tech aliment 23: 511-110.

23. Hashmi MS,Alam S, RiazA\&Shah A S(2007). Studies on microbial and sensory quality of mango pulp storage with 
chemical preservatives. Pak J of Nutr 6(1): 85-88.

24. Rodriqo D, Arranz JI, Koch S, Frigola, Rodriqo MC, Esteve MJ, Calvo C \& Radriqo M (2003). Physiochemical characteristics and quality of refrigerated spinach orange carrot juices and influence of storage conditions. $J$ Food $S c i$ 68(6):2111-2116.

25. Sarolia DK \& Mukarjee S (2002). Comparative efficiency of different preservation methods in keeping quality of lime (Citrus Aurantifolia) single juice during storage. Haryana J Hort Sci 31(34):185-188.

26. Zeb A, Ahmed I \& Ayub M (2009). Grape juice preservation with benzoate and sorbate. J Adv food Sci 31(1):17-21.

27. Ayub M \& Bilal KM (2001). Effect of different light conditions and colored glass bottles on the relation of quality characteristics of pomegranate syrup during storage at room temperature. Sarhad JAgric 17(4):629-632.

28. Wisal S, Zeb A, Ayub M \&Ullah I (2013). Refrigeration storage studies of strawberry juice with tss of 7.5 and 20.5 brix treated with sodium benzoate and potassium sorbate. Sarhad J Agric 29:3.

29. Kumar SP, Sagarand VR (2008).Quality of osmovac dehydrated ripe mango slices influenced by packaging material and storage temperature. J Sci Industries Research 67:1108-1114.

30. Zia A, Ayub M (2012). Quality Evaluation of melon cubes preserved in sweetner solution. MSc thesis. Food Sci Tech Deptt Uni of Agric,Peshawar.

31. Ghoria K \& Khurdiya DS (1998). Storage of heat processed Kinnow (Mandarin) Juice. J of Food Sci\& Tech 35(5):422-424.

32. Brenndor, Kennedy CO. Oswin DS, Trim GC, Mrema \& Werek C (1985). Solar driers and their role in post-harvest processing. Common Wealth Sci Council 78-83.

33. Heikal HA \& Sidawi MH (1988). Some factors responsible for the browning of lime and orange juices. Agric Res Rev 50(5):199-214.

34. Jain S, Shankla APK, Dashora A \& Shankla AK (2003). Physiochemical and sensory properties of orange drink. J Food Sci Tech India 40(6):656-659.

35. Bezman Y, Russell L, Rouseff\&Naim M (2001). 2-Methyl-3-furanthiol \&methional are possible off-flavours in nstored orange juice. J Agric Food Chem 49(5): 425-432.

36. Shimoda M \& Osajima Y (1981). Studies on off flavor formed during storage of Satsuma mandarin juice. J Agric Chem 55(4):319-324.

37. Okilya S, Muskiya IM \& Kaaya AN (2009). Effect of solar drying on the quality and acceptability of jack fruit leather. Electron J Env Agri Food chem. 9:101-111.

38. Nelugen SE \& Mahendran T (2010). Preparation of ready to serve (RTS) baverages from palmyrah (BorassusflabeliferL) fruit pulp. J Agric Sci 5(2):234-237.

39. Sharma SK, Chaudary SP, Rao VK, Yadav VK \&Bisht TS (2013). Standardization of technology for preparation and storage of wild apricot fruit bar. J Food Sci Tech 50:784-790.

40. Khan SH, Khan A, Litaf U, Shah AS, Khan MA, Ali MS, Shah FN, Maqbool Z, Adnan M \& Rani S (2015). Effect of different concentrations of guava pulp, apple pulp and sugar solution on the shelf stability of blend leather storage at ambient temperature. J Food Process Technol 6:7.

41. Shah W, Sufi NA \& Zafar SI (2008). Studies on the storage stability of guava fruit juice. Pak J Sci 18(3-4): 179-183. 